\title{
MOMENTO ANESTÉSICO-CIRÚRGICO: TRANSITANDO ENTRE O CONHECIMENTO DOS(AS) ENFERMEIROS(AS) E O CUIDADO DE ENFERMAGEM
}

\author{
Applying anesthesia during surgery: an exchange \\ between nursing knowledge and care
Momento anestésico y quirúrgico: transitando entre el conocimiento de los(las) enfermeros(as) y el cuidado de enfermería \\ Tania Marisa Koch', Denise Consuelo Moser Aguiar², Gelson Aguiar da Silva Moser², \\ Marceli Cleunice Hanaue ${ }^{3 *}$, Dulcimar de Oliveira ${ }^{3}$, Suellen Rodrigues de Oliveira Maier ${ }^{4}$
}

RESUMO: Objetivo: Verificar o conhecimento dos enfermeiros(as) de um hospital público da região oeste do estado de Santa Catarina sobre o cuidado de enfermagem no momento anestésico-cirúrgico. Método: Estudo de campo, descritivo-exploratório, qualitativo, utilizando-se entrevista semiestruturada com sete enfermeiros, observação e registros em diário de bordo. Resultados: Os resultados foram categorizados em conhecimento e cuidado e demonstram que os profissionais estão preocupados com o fazer, minimizando o cuidado humanizado e individualizado. Observou-se que a essência do cuidar reluta em emergir e permanecer integrada ao conhecimento científico. Conclusão: Percebemos que alguns enfermeiros não exercem funções prioritárias, entrando na rotina de trabalho, de modo a seguir normas e regras.

Palavras-chave: Anestesia. Enfermagem perioperatória. Cuidados de enfermagem.

ABSTRACT: Objective: To check the knowledge of nurses with regard to nursing care at the moment of applying anesthesia during surgery at a public hospital in the western region of the state of Santa Catarina. Method: A descriptive-exploratory and qualitative field study that performed semi-structured interviews with seven nurses, and used observation and logbook records. Results: The results were categorized into knowledge and care and they demonstrated that nursing professionals are concerned with both, thus minimizing humanized and individualized care. It was observed that the essence of caring is difficult to integrate with scientific knowledge. Conclusion: It was found that some nurses do not perform essential functions, for they enter into a work routine in order follow norms and rules.

Keywords: Anesthesia. Perioperative nursing. Nursing care.

RESUMEN: Objetivo: Verificar el conocimiento de los(las) enfermeros(as) de un hospital público de la región oeste del estado de Santa Catarina sobre el cuidado de enfermería durante el momento anestésico y quirúrgico. Método: Estudio de campo, descriptivo y exploratorio, cualitativo, utilizándose entrevista semiestructurada con siete enfermeros, por medio de observación y registros en cuaderno de bitácora. Resultados: Los resultados fueron categorizados en conocimiento y cuidado y demuestran que los profesionales están preocupados con el hacer, minimizando el cuidado humanizado e individualizado. Se observó que la esencia del cuidar resiste en emerger y permanecer integrada al conocimiento científico. Conclusión: Percibimos que algunos enfermeros no ejercen funciones prioritarias y por lo tanto entran en la rutina de trabajo de modo a seguir normas y reglas.

Palabras clave: Anestesia. Enfermería perioperatoria. Atención de enfermería.

\footnotetext{
'Enfermeira, Universidade Federal da Fronteira Sul (UFFS) - Chapecó (SC), Brasil.

2Doutores; Professores do curso de graduação em enfermagem, UFFS - Chapecó (SC), Brasil.

${ }^{3}$ Graduanda em enfermagem do $10^{\circ}$ período, UFFS - Chapecó (SC), Brasil.

4Mestre, Professora do curso de graduação em enfermagem, UFMT - Rondonópolis (MT), Brasil.

*Autor correspondente: tilihanauer@hotmail.com

Recebido: 28/08/2017 - Aprovado: 03/12/2017

DOl: 10.5327/Z1414-4425201800010003
} 


\section{INTRODUÇÃO}

Considera-se centro cirúrgico (CC) uma unidade de alta complexidade com tecnologias e procedimentos que invadem a privacidade dos pacientes. Assim, a importância da individualidade no atendimento e da humanização dos enfermeiros envolvidos nesse processo exigem do profissional de enfermagem amplo conhecimento sobre o período perioperatório, especialmente em relação ao momento anestésico-cirúrgico.

O tempo de permanência reduzido do paciente no CC não exclui a necessidade de uma assistência multiprofissional e de enfermagem com excelência e qualificação. A Sistematização da Assistência de Enfermagem Perioperatória (SAEP) objetiva a segurança do paciente e da equipe envolvida no ato operatório. A equipe de enfermagem deve estabelecer e desenvolver estratégias de ações no cuidado ao paciente pré-cirúrgico, sendo estas desenvolvidas conforme as particularidades de cada procedimento cirúrgico ao qual o paciente será exposto. Os cuidados devem ser executados segundo conhecimento especializado, para, assim, atender às necessidades decorrentes do procedimento realizado ${ }^{1}$.

A SAEP objetiva, principalmente, garantir uma assistência planejada em todo o período perioperatório, que acontece desde o pré-operatório (quando o paciente é informado da necessidade de realizar um procedimento cirúrgico e hospitalização), o intraoperatório (cirurgia propriamente dita) e o pós-operatório (após a cirurgia, período de recuperação). No pós-operatório, o paciente pode apresentar complicações decorrentes do ato anestésico-cirúrgico, uma vez que elas podem estar associadas a um preparo pré-operatório inadequado. A partir de 2002, a SAEP se tornou uma exigência do Conselho Federal de Enfermagem (COFEn), para auxiliar as equipes de enfermagem e médica, e todos os demais envolvidos a garantir uma assistência segura ao paciente ${ }^{2}$.

O enfermeiro é o profissional apto a coordenar todas as etapas do período perioperatório, possibilitando ambiente seguro, adequado, asséptico no decorrer do ato anestésicocirúrgico, tanto ao paciente como também à própria equipe. Nesse contexto, o enfermeiro deve estar atento a todas e quaisquer reações que o paciente possa apresentar ${ }^{1}$.

Sugere-se a aplicação da SAEP como estratégia para proporcionar um cuidado integral e individualizado, de modo a auxiliar paciente e família a conhecerem e compreenderem todo o processo que será realizado, tranquilizando-os quanto aos riscos aos quais o paciente poderá ser exposto. Para isso, são preconizadas visitas pré e pós-operatória, com o intuito de qualificar a sistematização. Nesse contexto, insere-se, também, a orientação, o preparo físico e emocional, a avaliação e o encaminhamento ao CC, a fim de diminuir riscos e promover uma recuperação mais efetiva, considerando a dependência e/ou a vulnerabilidade de cada pessoa ${ }^{3,4}$.

A insegurança do paciente independe da complexidade da cirurgia, tendo relação direta com a desinformação quanto aos procedimentos cirúrgicos, à anestesia e aos cuidados realizados no pós-operatório. A ação educativa faz parte da atribuição do enfermeiro no período perioperatório, devendo desenvolver suas condutas junto ao paciente no pré-operatório, de modo que lhe transmita segurança e tranquilidade. Nesse contexto, enquadram-se duas ferramentas essenciais do cuidado, a comunicação e interação com o paciente, que conduzirão a uma cautela específica, de acordo com as necessidades e expectativas do paciente cirúrgico ${ }^{4}$.

Durante as atividades teórico-práticas no CC, surgiram inquietações e indagações no que se refere ao cuidado prestado pelo profissional enfermeiro. Dessa forma, a pergunta norteadora da presente pesquisa foi: qual o conhecimento dos profissionais enfermeiros sobre o cuidado de enfermagem no momento anestésico-cirúrgico?

Na tentativa de nortear e percorrer as possibilidades que pudessem responder essa questão, buscou-se na literatura científica e na pesquisa de campo informações sobre os elementos que constituem o conhecimento dos profissionais enfermeiros e os cuidados de enfermagem realizados no momento anestésico-cirúrgico e na recuperação pós-anestésica (RPA).

\section{OBJETIVO}

Verificar o conhecimento dos enfermeiros(as) sobre o cuidado de enfermagem no momento anestésico-cirúrgico em um hospital público de Santa Catarina.

\section{MÉTODO}

O presente estudo foi realizado no CC de um hospital público da região oeste do estado de Santa Catarina. Os protagonistas do estudo foram os enfermeiros envolvidos no momento anestésico-cirúrgico do referido hospital, que concordaram em participar da pesquisa. O serviço contou com a presença de sete profissionais de enfermagem de nível superior: um enfermeiro coordenador; dois enfermeiros assistenciais que atuam na sala de RPA; e quatro enfermeiros assistenciais no $\mathrm{CC}$, sendo que dois deles trabalham no período noturno. 
Após aprovação do Comitê de Ética em Pesquisa da Universidade Federal da Fronteira Sul, por meio do Parecer $n^{\circ} 785613$ e da autorização da instituição hospitalar sede do estudo, elaborou-se o termo de consentimento livre e esclarecido (TCLE), que foi entregue aos participantes da pesquisa para assinatura. Os enfermeiros foram convidados a participar do estudo espontaneamente. Realizou-se contato inicial com os profissionais da devida unidade e foram estabelecidos e agendados dois a três encontros, com duração média de 20 a 30 minutos.

No primeiro momento foram feitas as entrevistas com questões semiestruturadas, as quais foram gravadas e, em seguida, transcritas na íntegra. No segundo momento da pesquisa utilizou-se a observação a partir de um registro em forma de diário de bordo. Isso ocorreu entre a segunda quinzena de setembro de 2014 e outubro do mesmo ano. Após a realização das etapas de coleta, deu-se início à organização do material para análise dos dados, a qual foi concretizada com base no exame de conteúdo, proposto por Bardin. Para possibilitar a identificação das falas dos entrevistados mantendo o sigilo, foram adotados codinomes, baseados em escalas (Ramsey, Richmond, Jouvet, Steward, Mallampatti, Cromacke Lehane) aplicadas ao paciente cirúrgico.

\section{RESULTADOS}

Após análise e codificação dos dados, por meio das entrevistas e observações realizadas na instituição, obtiveram-se duas categorias, as quais foram destacadas e reunidas conforme os Quadros $1 \mathrm{e} 2$.

O primeiro quadro apresenta as questões referentes ao conhecimento dos enfermeiros da unidade sobre o momento anestésico-cirúrgico relacionado ao cuidado de enfermagem, atentando para a forma de conduzir o olhar para o outro de forma humanizada, afetuosa e cuidadora. Nesse sentido, mais duas questões se colocam: no que se constitui o cuidado para além da assistência técnica? É possível realizar uma assistência humanizada, especializada e individualizada baseada em teorias, considerando o conhecimento técnico-científico?

O Quadro 2 apresenta as questões que permeiam o cuidado e como ele é evidenciado pelos(as) enfermeiros(as) durante a assistência de enfermagem ao paciente no momento anestésico-cirúrgico.

\section{DISCUSSÃO}

Sob a influência de Florence Nightingale, a enfermagem iniciou a sua caminhada para a utilização de uma prática baseada
Quadro 1. Codificação dos dados da primeira categoria de análise. Primeira categoria de análise: conhecimento

0 conhecimento no percurso do cuidado: um movimento possível

Pergunta de pesquisa: Qual o conhecimento dos profissionais enfermeiros(as) sobre o cuidado de enfermagem no momento anestésico-cirúrgico?

Enf. Ramsey: "O paciente é inicialmente sedado, depois intubado, logo após extubado e encaminhado para a sala de recuperação."

Enf. Richmond: "Início ocorre a intubação, na sequência então a sedação, e a parte final é acordar o paciente e extubá-lo."

Enf. Steward: "Esse processo pode ser divido em fases, a primeira chamada de indução, depois a manutenção, e a recuperação."

Quadro 2. Codificação dos dados da segunda categoria de análise. Segunda categoria de análise: cuidado

0 cuidado desvelado na assistência prestada: entrelaçando potências e possibilidades

Pergunta de pesquisa: Qual o conhecimento dos profissionais enfermeiros(as) sobre o cuidado de enfermagem no momento anestésico-cirúrgico?

Enf. Cromack: "Na recuperação anestésica recebe o paciente, entrevista se for possível, se não for ler as anotações e o correto seria prescrever um plano de cuidados, mas isso não ocorre."

Enf. Steward: “Proporcionar conforto ao cliente, monitorização, sinais vitais, oxigenação, administrar medicamentos conforme prescrição médica."

Enf. Lehane: “Quando é possível, usa-se a teoria holística e também o processo de enfermagem".

Enf. Mallampatti: “Assistir o paciente, transmitir e passar tranquilidade, confirmação de jejum e alergias do paciente, cuidar com a exposição do corpo do paciente. Cuidar com a manutenção da temperatura, realizar um checklist de cirurgia segura, utilizar os equipamentos apropriados conforme avaliação do paciente."

Enf. Richmond: “(...) quando o paciente chega na sala de recuperação, no primeiro momento, independentemente do tipo de cirurgia ou anestesia que o paciente fez, deve-se instalar um oxigênio, principalmente para prevenir a hipóxia (...), o paciente chega meio sonolento, então às vezes eles acabam esquecendo de respirar, então é importante colocar um $\mathrm{O}_{2} \ldots$ A monitorização, controle dos sinais vitais, prevenir então a hipotermia, aquecendo o paciente. Cuidar (...) de náuseas, vômitos, ficar atento para o paciente não broncoaspirar... E os sinais vitais, que seria a monitorização contínua... Formulário de admissão do paciente, primeiro momento coloca os dados do paciente, depois faz um checklist... E daí tem uma escalinha que são verificados então os sinais vitais... A enfermeira então, eu faço a escala de Aldrete a hora que ele chega e depois a hora quando eu vejo que ele está bem recuperado para pegar a alta e esse documento vai junto com o paciente no prontuário." 
em evidências ou conhecimentos científicos, abandonando gradativamente a postura de atividade bondosa, intuitiva e/ou experiências vividas. Nesse aspecto, diversos conceitos, teorias e modelos específicos à ciência da enfermagem foram e estão sendo desenvolvidos, direcionados à necessidade de prestar uma assistência sistematizada, permitindo qualificar o atendimento prestado ao paciente no momento anestésico-cirúrgico de forma holística.

Observa-se, atualmente, o trabalho da enfermagem desenvolvido no CC focado na realização do ato anestésico-cirúrgico, o qual consiste em providenciar os materiais, equipamentos e recursos humanos necessários para sua realização, distanciandose do cuidado e da assistência voltada ao atendimento das necessidades do paciente e de seu bem-estar, a partir da visão integrada, preservando sua integridade, conforme a teoria Levine.

Quando questionados sobre o processo anestésico e suas etapas, os enfermeiros descrevem como ocorre essa etapa, mas não mencionam o cuidado de enfermagem para com o paciente.

“... O paciente é inicialmente sedado, depois intubado, logo após extubado e encaminhado para a sala de recuperação." (Enf. Ramsey)

“... No início ocorre a intubação, na sequência então a sedação, e a parte final é acordar o paciente e extubá-lo ..." (Enf. Richmond)

“... Esse processo pode ser dividido em fases, a primeira chamada de indução, depois manutenção, e a recuperação ..." (Enf. Steward)

Há uma preocupação relevante e destacada no que tange aos aspectos técnicos da assistência. Percebe-se a realização de ações rotineiras, cadenciadas e sistemáticas no processo de assistência ao paciente durante o momento anestésico, rotina essa que parece consumir os enfermeiros, tanto pela carga de trabalho quanto pelo déficit de recursos humanos. Observa-se que a atuação profissional, muitas vezes, ainda se mostra autoritária, preocupada em seguir normas e rotinas, com aspectos formais e objetivos, embora se saiba que o vínculo no cuidar busca conhecer as reais necessidades do paciente e planejar uma assistência de enfermagem de qualidade, humanizada e individualizada. Destaca-se que alguns profissionais atentam às necessidades que surgem em decorrência dessa dinamicidade e buscam associar o conhecimento técnico-científico ao cuidado de enfermagem.

O pré-operatório é definido como um período interativo para detecção das reais necessidades físicas e psicológicas, as quais auxiliam no planejamento do cuidado. Tal assistência demanda habilidade e conhecimento a respeito das prováveis reações, emocionais ou fisiológicas que o paciente pode apresentar diante da anestesia e da cirurgia. Logo, atingirá objetivos traçados no plano de cuidados, promovendo a integridade e a plenitude biopsicossocioespiritual ${ }^{5}$.

A orientação pré-operatória exige do enfermeiro responsável uma ação assídua, que se torna parte do exercício profissional. Pela orientação, o profissional de enfermagem pode atuar promovendo um espaço de acolhimento ao paciente e também à sua família, estabelecendo vínculos, além da orientação em si, e atuando como educador em saúde.

No que se refere à assistência de enfermagem prestada na RPA, o enfermeiro pode lançar mão de protocolos do gerenciamento de riscos, que corresponde às instruções de boas práticas assistenciais no intuito de prevenir determinado risco ou evento adverso mapeado, garantindo maior segurança ao paciente na sala de RPA. Nesse processo, ressalta-se a importância de olhar para o paciente como um ser humano que necessita de cuidados, considerando que o processo de cuidar não consiste apenas em gerir e aplicar processos técnicos, normas e rotinas, mas, também, um olhar humanizado e acolhedor, ser compreendido como ser humano em todas as suas dimensões.

“... Na recuperação anestésica, recebe o paciente, entrevista se for possível, se não for, lê as anotações e o correto seria prescrever um plano de cuidados, mas isso não ocorre ...” (Enf. Cromack)

Compete ao enfermeiro a adoção de diretrizes que foquem em segurança e qualidade na RPA, prevenção de eventos adversos, caminhando de acordo com as políticas de segurança ao paciente. Nessa lógica, a SAEP constitui uma ferramenta importante de raciocínio clínico, visando a auxiliar o enfermeiro na clareza das necessidades durante o momento anestésico-cirúrgico, pois o paciente, quando se encontra em um ambiente de alta complexidade e com acesso restrito, necessita de atendimento diferenciado, específico e qualificado da equipe de profissionais cirúrgicos ${ }^{1}$.

Destaca-se, ainda, que a utilização da SAEP é um método científico e específico do enfermeiro, que proporciona o gerenciamento e o aperfeiçoamento da assistência prestada, de maneira organizada, segura, dinâmica e competente. Também confere cientificidade ao desenvolvimento da enfermagem como profissão e auxilia no seu crescimento, o que implica o fazer acompanhado do saber, com desenvolvimento 
de pensamento crítico. Além disso, é uma ferramenta que promove soluções de problemas e tomada de decisões, melhorando o reconhecimento profissional, o que, muitas vezes, serve de estímulo aos trabalhadores ${ }^{6}$.

Essas mudanças podem se aproximar de uma prática de enfermagem mais organizada, possibilitando a implantação da SAEP como instrumento importante de trabalho do enfermeiro ${ }^{3}$.

A importância do enfermeiro na participação de programas educativos, na avaliação e no controle da dor é efetiva, por ser este profissional que permanece mais tempo ao lado do paciente. Assim, deve utilizar esse contato para identificar as demandas de mudanças nos métodos de analgesia, providenciar ajustes, quando necessários, e educar/orientar o paciente e seus familiares sobre a dor, por ser um dos principais efeitos indesejados no pós-operatório. Na visão do paciente, essa é uma perspectiva importante no controle da qualidade da assistência prestada na sala de RPA. Quando esse efeito ocorre, além de métodos farmacológicos, o enfermeiro deve implementar terapias que aliviem a dor, como aplicação de frio ou calor no local, massagens, uso de coxins, mudança de posição e comunicação, fortalecendo o vínculo enfermagempaciente, promovendo um cuidado integralizado, individualizado e melhora da qualidade de vida do paciente com $\operatorname{dor}^{7-9}$.

No período de permanência do paciente na RPA, sabe-se que ele está exposto a alguns riscos clínicos, como depressão respiratória, instabilidade cardiovascular, incapacidade de deambulação, rebaixamento do nível de consciência, náuseas e vômitos, hipotermia, sangramentos, retenção urinária e erros de medicações. Compete ao profissional enfermeiro gerenciar e minimizar a incidência desses riscos.

A SAEP visa à utilização de uma metodologia de trabalho, independente do referencial teórico utilizado, e requer do enfermeiro interesse em conhecer o paciente como indivíduo, utilizando seus conhecimentos e habilidades, além de orientação e treinamento da equipe de enfermagem para a implementação das ações sistematizadas. Logo, a enfermagem articula e encaminha todos os processos que resultam no cuidado e, nesse sentido, acredita-se que o conhecimento desse percurso seja um movimento possível.

A rotina e o cotidiano, tão citados como muros que limitam alguns movimentos, também podem ser espaços de criatividade, já que na rotina descobrimos formas de fugir das normas e das convenções. É importante salientar que destacar as dificuldades e dizer o que sabemos não nos leva a criar outras formas de cuidar e de nos aproximar do cuidado; para tanto, há que se pensar em estratégias possíveis de reinventar a arte de cuidar, que pode ser vivida com toque, afeto, carinho e respeito com e pelo outro.

Nesse sentido, estima-se que o enfermeiro e sua equipe desenvolvam competências específicas para cuidar desse indivíduo de modo humanizado e acolhedor, o que requer sensibilidade suficiente para atender o paciente cirúrgico em todos os seus aspectos. Alguns profissionais chegam a citar o processo de enfermagem; porém, por alguns motivos, não o utilizam rotineiramente. A SAEP, por ser um ótimo instrumento, também exibe outros métodos de cuidado que auxiliam na qualificação do cuidar, citando a metodologia da assistência de enfermagem (MAE) e o processo de enfermagem $(\mathrm{PE})^{9}$.

"Quando possível usa-se a teoria holística e também o processo de enfermagem ..." (Enf. Lehane)

Pode-se considerar que o cuidado, mesmo quando guiado pelo processo de enfermagem, não se circunscreve de forma estanque em suas etapas, pois ele é um constante ir e vir dentro da relação que se estabelece entre o enfermeiro e o paciente, principalmente no CC, por ser uma unidade extremamente complexa. Esse movimento é atribuído não só ao paciente e à sua condição, mas, também, à sofisticação de equipamentos e tecnologias avançadas, ao elevado volume de informação que veicula, à natureza da comunicação e à coordenação da equipe.

Tais aspectos, associados à fadiga, ao stress, às pressões de produção e a pesadas cargas de trabalho, fazem com que o CC se torne um local vulnerável a eventos adversos. Os eventos adversos cirúrgicos podem ocorrer devido à comunicação deficiente entre a equipe, má técnica cirúrgica do cirurgião e elementos da equipe, mau funcionamento ou utilização inadequada dos equipamentos, e agravados por problemas de recursos e organização. Em meio a tudo isso, encontramos o profissional enfermeiro que deve preservar e garantir uma assistência de enfermagem com qualidade, segurança, humanizada e acolhedora. Para tanto, a utilização do processo de enfermagem é um instrumento de suma importância, obtendo objetivos traçados.

Busca-se o comprometimento dos enfermeiros em oferecer aos pacientes cirúrgicos uma assistência de enfermagem diferenciada. Os objetivos dessa forma de cuidar visam prevenir complicações decorrentes do momento anestésicocirúrgico. O que pode ser evidenciado pela fala dos participantes, que ressaltam a importância de assistir o paciente, transmitir tranquilidade, proporcionar conforto e o cuidado com a exposição do corpo: 
“Proporcionar conforto ao cliente...” (Enf. Steward)

“...Assistir o paciente, transmitir e passar tranquilidade [...] cuidar com a exposição do corpo do paciente, utilizar os equipamentos apropriados conforme avaliação do paciente. Cuidar da manutenção da temperatura, realizar um checklist de cirurgia segura ..." (Enf. Mallampatti).

Outro fator que pode auxiliar e ser benéfico é a realização da visita pré-operatória de enfermagem, que é considerada uma ferramenta eficaz e potencializadora do cuidado durante o momento anestésico-cirúrgico. Pode ser útil na promoção do cuidado individualizado, na obtenção de dados sobre a personalidade e as características físicas e emocionais do paciente, bem como auxilia na recuperação pós-cirúrgica.

A visita pré-operatória pode ser conduzida de tal forma que minimize a tensão, o medo e a ansiedade, tornando-se bastante benéfica ao paciente. Ela visa a proteger todas as partes envolvidas no processo de cuidar, proporcionando bem-estar ao paciente, promovendo visibilidade do cuidado ao profissional enfermeiro e fornecendo subsídios ao planejamento da assistência, de forma contínua e individualizada.

Nesse contexto, após a publicação pelo Ministério da Saúde (MS) do Programa Nacional de Segurança do Paciente (PNSP), buscando a prevenção de eventos adversos nos serviços de saúde, a visita pré-operatória passou a ter maior destaque e relevância nas unidades cirúrgicas. No entanto, o que se observa ainda na prática diária é a presença de alguns obstáculos para a execução dessa etapa ${ }^{10}$.

Em estudo realizado ${ }^{9}$, destaca-se a importância da enfermagem na assistência prestada na sala de recuperação anestésica (SRA), por ter como meta assistir, monitorar, prevenir complicações, garantir a segurança e contribuir ao máximo para o bem-estar do paciente. Os mesmos autores consideram o período pós-operatório imediato, na RPA, como crítico, uma vez que o paciente passa por um procedimento cirúrgico e recebe medicações anestésicas, exigindo, assim, vigilância constante da equipe de enfermagem. É essencial prestar uma assistência integral ao paciente e sempre registrá-la no prontuário. Os cuidados devem ser realizados de forma adequada, impedindo a ocorrência de complicações e/ ou de eventos adversos. Esses aspectos podem ser observados na fala dos pesquisados:

“... Instalar oxigênio principalmente para prevenir a hipóxia ... monitorização, então controle dos sinais vitais, prevenir então a hipotermia, aquecendo o paciente. Cuidar em relação então a questão de náuseas, vômitos, ficar atento para o paciente não broncoaspirar... eu faço a escala de Aldrete." (Enf. Richmond)

Existe grande necessidade de os profissionais de enfermagem buscarem conhecimento após concluírem sua formação profissional, para que estejam atualizados, pois isso sustenta a qualidade da sua prática e a segurança dos pacientes. Os enfermeiros buscam diariamente formas de enfrentar as barreiras operacionais da profissão, na tentativa de um cuidado qualificado. É necessário desenvolver ações desde o processo de formação dos enfermeiros, partindo da vida acadêmica, plantando a semente do cuidado que toca, que cuida, que cura e que vive.

\section{CONSIDERAÇÕES FINAIS}

Notou-se, por meio deste estudo, competências necessárias ao enfermeiro, descrevendo suas atividades no dia a dia nas quatro esferas (gestão, pesquisa, ensino e assistência). Assim, evidenciou-se que ainda existem lacunas sobre o tema, permitindo avaliar o conhecimento dos profissionais envolvidos no processo do cuidado no $\mathrm{CC}$ e na RPA, quanto ao momento anestésico-cirúrgico, principalmente no que diz respeito à anestesia e ao conhecimento quanto à assistência de enfermagem prestada ao paciente anestesiado.

Entende-se a importância de a enfermagem proporcionar boas práticas para garantir bons resultados, com o intuito de se propiciar um cuidado humanizado, acolhedor e de qualidade, com sua atenção focada no paciente e não apenas na doença, ou em uma parte do seu corpo.

Escolher ser um profissional da área da saúde demanda habilidades técnicas em relação a equipamentos e procedimentos pertinentes, conhecimentos científicos, capacidade de dialogar, perceber, vivenciar e ver o paciente como um todo. Apesar de muitas instituições hospitalares ainda não adotarem totalmente esse processo de cuidar e sistematizar, a SAEP é vista como um método articulador e integrador da assistência de enfermagem no momento anestésico-cirúrgico, bem como na continuação desse cuidar.

Finalizamos destacando que o momento anestésico-cirúrgico é singular para o paciente que vivencia o procedimento e para sua família. A pesquisa aqui descrita e apresentada permitiu transitar entre o conhecimento dos enfermeiros e o cuidado de enfermagem, de maneira a lançar muitas reflexões para a vida profissional dos que atuam na enfermagem perioperatória. 


\section{REFERÊNCIAS}

1. Associação Brasileira de Enfermeiros de Centro Cirúrgico, Recuperação Anestésica e Centro de Material de Esterilização (SOBECC). Práticas recomendadas SOBECC. 6a ed. São Paulo: Manole; 2013.

2. Monteiro EL, Melo CL, Amaral TL, Prado PR. Cirurgias seguras: elaboração de um instrumento de enfermagem perioperatória. Rev SOBECC. 2014;19(2):99-109.

3. Adamy EK, Tosatti M. Sistematização da assistência de enfermagem no período perioperatório: visão da equipe de enfermagem. Rev Enferm UFSM. 2012;2(2):300-10.

4. Camponogara S, Soares SG, Silveira M, Viero CM, Barros CS, Cielo C. Percepção de pacientes sobre o período pré-operatório de cirurgia cardíaca. Rev Min Enferm. 2012;16(3):382-90.

5. Amthauer C, Falk JW. O enfermeiro no cuidado ao paciente cirúrgico no período pré-operatório. Rev Enferm [Internet]. 2014 [acesso em 2017 dez. 08];10(10):54-9. Disponível em: http://www.revistas.fw.uri. br/index.php/revistadeenfermagem/article/view/1386/1849

6. Ramos AS, Pereira EB, Silva GW, Lira JS, Fernandes LC. Construção de uma cartilha educativa como ferramenta de apoio à sistematização da assistência de enfermagem perioperatória e à experiência cirúrgica: relato de experiência. Rev Extensão da UFMG. 2016;4(1):173-81.

7. Nascimento JC, Silva LC. Avaliação da dor em pacientes sob cuidados em unidades de terapia intensiva: uma revisão de literatura. Rev Movimenta [Internet]. 2014 [acesso em 2017 dez. 08];7(2):711-20. Disponível em: http://www.revista.ueg.br/index.php/movimenta/ article/view/6274/4322

8. Popov DC, Peniche AC. As intervenções do enfermeiro e as complicações em sala de recuperação pós-anestésica. Rev Esc Enferm. 2014;43(4):953;61. DOI: 10.1590/S0080-62342009000400030

9. Macena MD, Zeferino MG, Almeida DA. Assistência do enfermeiro aos pacientes em recuperação pós-cirúrgica: cuidados imediatos. Rev Iniciação Científica Libertas [Internet]. 2014 [acesso em 2017 dez. 08];4(1):133-51. Disponível em:http://www.libertas.edu.br/revistas/ index.php/riclibertas/article/view/52

10. Brasil. Ministério da Saúde. Portaria n ${ }^{\circ} 529$, de $1^{\circ}$ de abril de 2013. Institui o Programa Nacional de Segurança ao Paciente (PNSP). Brasília; 2013. 